\title{
Representación social de la inmigración en el discurso del Partido Popular
}

\author{
GEMA RUBIO CARBONERO \\ UNIVERSITAT POMPEU FABRA
}

ABSTRACT: This article explores the social representation of immigration that emanates from Partido Popular's political discourse. Partido Popular (PP) is a political party which was at the head of the state in Spain from 1996 to 2004. From Critical Discourse Analysis perspective, we will carry out a semantic analysis of the main themes and macropropositions that compose the macrostructure of the selected corpus. This corpus is formed by twenty-four parliamentary debates and thirty-two interviews done to different members of Partido Popular about immigration. From this analysis, we will show how underlying ideologies operate, how they appear discursively and they determine the speaker's linguistic production. In the same way, we will make explicit the forms by which Partido Popular's discourse constructs and reconstructs prejudices against migrants.

Keywords: Political discourse, Partido Popular, immigration, social representation, prejudices, CDA.

RESUMEN: este artículo explora la representación social de la inmigración que se desprende del discurso político del Partido Popular. El Partido Popular (PP) es un partido político que estuvo a la cabeza del gobierno en España entre los años 1996 y 2004. Desde la perspectiva del Análisis Crítico del Discurso, se lleva a cabo un análisis semántico de la temática y las macroproposiciones que componen la macroestructura del corpus seleccionado. Este corpus está compuesto por veinticuatro debates parlamentarios y treinta y dos entrevistas realizadas a distintos miembros del Partido Popular sobre inmigración. Este análisis desvelará cómo operan las ideologías subyacentes y cómo estas se manifiestan discursivamente, determinando la producción lingüística de los hablantes. Igualmente, se harán explícitas aquellas formas de proyección, construcción y reconstrucción de prejuicios sobre las personas migradas, que se desprenden del discurso del Partido Popular sobre inmigración.

Palabras clave: discurso político, Partido Popular, inmigración, representación social, prejuicios, ACD. 


\section{Introducción}

Uno de los temas que ha incrementado en relevancia en la agenda política española durante los últimos años es el de la inmigración. Tanto es así, que Zapata-Barrero, et al (2008) hablan de una politización de la inmigración. Como ejemplo ilustrativo, basta mencionar que, entre los años 1992 y 1996, se detectaron en el congreso de los diputados de España 71 iniciativas que trataban sobre la inmigración. Entre 1996 y el año 2000, se recogieron 574 y, entre los años 2000 y 2004, se detectaron un total de 2083 iniciativas.

El importante papel que juegan los políticos en la representación social de esta realidad es innegable. Tanto los políticos, como los medios de comunicación, tienen una muy alta responsabilidad en la creación de actitudes y opiniones de la sociedad hacia un fenómeno como el de la inmigración. En este artículo, queremos acercarnos a esta representación social y, en concreto, a los roles y atributos que se asignan a las personas migradas a través del discurso político. En esta búsqueda de la representación social (Moscovici, 1979, 1986, 2001) de las personas migradas, focalizaremos especialmente en la proyección de posibles prejuicios y/o actitudes negativas hacia estas personas.

Para este fin, se ha llevado a cabo un estudio sobre el discurso político del Partido Popular sobre inmigración. El Partido Popular (PP), entre los años 1996 y 2004, fue el partido que estuvo a la cabeza del gobierno en España. Fue, precisamente, durante la primera legislatura del PP (entre 1996 y el 2000) cuando se aprobó la segunda gran reforma de la ley de extranjería: la Ley LO 4/2000, que fue apoyada por todos los partidos, excepto por el PP, que gobernaba con mayoría simple.

No obstante, cuando el PP consiguió la mayoría parlamentaria absoluta en las elecciones del año 2000, inmediatamente promulgó una nueva ley, que pudo aprobar gracias a esta mayoría parlamentaria: la Ley LO 8/2000. Esta ley se caracterizaba fundamentalmente por la distinción que se hacía entre inmigrantes de carácter legal e inmigrantes de carácter ilegal. A estos últimos, no se les reconocía ninguno de los derechos fundamentales de reunión, manifestación, sindicación, asociación o asistencia jurídica (Izquierdo, 2002). Esto fue, precisamente, uno de los puntos de esta nueva ley más criticados por el resto de los partidos políticos y por los medios de comunicación.

A pesar de ello, esta ley sentó las bases de nuestra actual ley de extranjería y el debate político y social en torno a la misma fue verdaderamente cuantioso. Por esta razón, consideramos que el Partido Popular, con la promulgación de esta ley, sentó igualmente las bases del conocimiento social sobre inmigración y jugó un papel vital en la representación social del fenómeno y de las personas migradas. En este artículo exploraremos, por tanto, la representación social de las personas migradas que se desprende del discurso del Partido Popular, entre los años 2000 y 2004. 
Para comenzar, se hace necesario indagar en el marco teórico sobre el cual se sustenta este estudio.

\section{El marco teórico}

Este estudio se encuadra dentro del Análisis Crítico del Discurso, que es una perspectiva crítica que se enmarca dentro de la disciplina general del Análisis del Discurso. Esta perspectiva supone un estudio multidisciplinario de las relaciones entre el discurso, el poder y la desigualdad social, en la que el investigador adquiere una actitud crítica sobre una determinada realidad social. Por tanto, se centra especialmente en cómo se representan discursivamente acontecimientos, relaciones sociales, y las implicaciones sociales de estos procesos de representación (Martín Rojo et al., 2003).

En particular, dentro de esta perspectiva, el modelo de análisis empleado será el enfoque socio-cognitivo enunciado y extensamente investigado por Van Dijk (1997, 1998, 2001, 2002, 2003, 2004, 2008). Este enfoque utiliza la cognición para mediar como una interfaz entre el discurso y la sociedad considerando, por un lado, la parte cognitiva de los emisores, para entender cómo esta da forma y condiciona la selección de las diferentes estructuras lingüísticas y, por otro lado, la parte cognitiva del oyente/lector.

De manera más concreta, Van Dijk (2008) sostiene que este enfoque considera no solo el conocimiento, sino también cómo el contexto, la ideología y las actitudes de los hablantes condicionan su producción lingüística, y cómo este discurso se almacena en la memoria de los oyentes/lectores e influye en la formación de sus opiniones, actitudes y, eventualmente, ideologías.

No disponemos del espacio suficiente en este artículo para adentrarnos en profundidad en las nociones de conocimiento, contexto, ideología, actitudes y opiniones. Baste con señalar que las ideologías se expresan y, por tanto, se adquieren a través del discurso. Igualmente, las ideologías controlan la identidad de los grupos y se manifiestan discursivamente a través de una estrategia ideológica general. Esta estrategia general, presenta una polarización entre nosotros (el endogrupo) y ellos (el exogrupo). En esta estrategia ideológica general, hay una tendencia a enfatizar nuestros aspectos positivos y sus aspectos negativos y, a su vez, a desenfatizar o mitigar nuestros aspectos negativos y sus aspectos positivos (para más detalles ver Van Dijk, $2001 b, 2002$ b, 2007).

Debido a las limitaciones de espacio, en este estudio nos centraremos en la representación global de la inmigración y, en concreto, en los posibles prejuicios, estereotipos y actitudes negativas hacia este colectivo que se desprenden del discurso del PP. 
Desde la psicología social, se han llevado a cabo numerosos estudios sobre la comprensión de qué son los prejuicios y los estereotipos y qué factores intervienen en la formación de los mismos. En particular, Allport (1977) define el prejuicio como una actitud hostil o desconfiada hacia una persona por el mero hecho de pertenecer a un grupo.

El estereotipo, por su parte, de acuerdo con Hamilton y Trolier (1986) es una estructura cognitiva que contiene conocimientos, creencias y expectativas sobre algún grupo humano.

Así, mientras el estereotipo (que puede ser positivo, negativo o neutro) es una simplificada representación generalizada de las propiedades atribuidas a una colectividad social (por ejemplo a una nación, un grupo étnico, etc.), el prejuicio es una actitud generalmente negativa hacia una persona o un colectivo social o situación que se basa en una información insuficiente o incompleta.

Para comprender qué prejuicios y que representación social sobre las personas migradas se desprenden del discurso del PP sobre inmigración, llevaremos a cabo un análisis detallado del contenido semántico global del corpus seleccionado. En otras palabras, indagaremos sobre la macroestructura, que es la estructura jerárquica global de significado semántico que se compone de un conjunto de proposiciones globales (macroproposiciones) que, a su vez, se derivan de los significados locales de las oraciones. Esta derivación se produce a través del uso de unas macroreglas, que son: la supresión, la generalización, la selección y la integración (Van Dijk, 1977).

Sabemos, gracias a la psicología cognitiva (Baddeley, 1999; Piaget, 1977), que el ser humano no puede recordar todos los detalles de un discurso concreto, sino que reduce la información semántica compleja de un discurso para almacenarlo en la memoria. Las macroestructuras son las estructuras del discurso que más efecto tienen en la construcción de los modelos mentales, que son representaciones mentales particulares y personales sobre un determinado evento (Van Dijk, 2008). Los modelos mentales se construyen a través de la información que se deriva del discurso conjuntamente con nuestro conocimiento general sobre un determinado tema. Estos modelos mentales son la base de la memoria de la gente y dan forma a las opiniones y las actitudes con respecto a un tema, puesto que definen cómo las personas interpretan cada situación.

Así, cuando leemos un texto, en la memoria a corto plazo no se puede almacenar toda la información para la producción y la comprensión de ese texto. Por el contrario, en lugar de mantener activas todas las microproposiciones que componen dicho texto, se produce una abstracción y una generalización que, de algún modo, resume el contenido semántico más importante a través de macroproposiciones que a nivel global ayudan a interpretar las microproposiciones de una manera coherente con el resto del discurso. Las macroproposiciones, consecuentemente, se construyen como modelos mentales coherentes de manera que 
se pone de manifiesto cuál es la información más importante para los participantes.

A su vez, estas macroproposiciones pueden expresar opiniones, por lo que tienen una clara influencia en la proyección social de estereotipos o prejuicios y, como consecuencia, en la formación de actitudes e ideologías particulares. Consecuentemente, el interés especial de las macroproposiciones reside, no solamente en el hecho de que definen la coherencia global del discurso, sino en que también representan la información mejor memorizada de un discurso y, por tanto, con más influencia sobre el público.

No obstante, en el sentido puramente estricto, una macroproposición es una proposición única. Sin embargo, nuestro análisis mostrará macroproposiciones generalizadas que se repiten constantemente a lo largo del corpus seleccionado. Por tanto, adoptaremos este término para referirnos a estas proposiciones generalizadas y recurrentes.

\section{Metodología}

\subsection{Descripción del corpus}

Para dar cuenta de los objetivos que plantea esta investigación, se han recolectado todos los discursos pronunciados por el Partido Popular sobre inmigración, en el Pleno y la Diputación Permanente entre el año 2000 y 2004. Igualmente, se han recogido todas las entrevistas a distintos miembros del Partido Popular, que aparecen en periódicos de ámbito nacional en el mismo periodo de tiempo.

La recolección de los discursos parlamentarios se ha realizado a través de la página del congreso de los diputados (www.congreso.es), accediendo y descargando todos los diarios de sesiones y buscando en ellos todos aquellos discursos en los que interviene algún miembro del PP hablando sobre algún aspecto de inmigración o extranjería. De esta recolección, surgieron cuarenta textos.

La recolección de las entrevistas políticas se ha realizado a partir de la base de datos Activa, que permite buscar todo tipo de archivos (artículos, entrevistas, noticias, etc.) publicados en los distintos periódicos de ámbito nacional. A partir de la selección del formato entrevista y la búsqueda de distintas palabras clave relacionadas con el PP y la inmigración, se obtuvieron más de cincuenta textos.

De esta primera recogida de textos, se produjo una lectura exhaustiva y se seleccionaron aquellos en los que la inmigración era el tema central del debate o de la entrevista y se descartaron aquellos que trataban la inmigración de manera tangencial. Así, se obtuvieron un total de treinta y dos entrevistas y veinticuatro debates parlamentarios. 
Puesto que el objetivo general de nuestra investigación es valorar el alcance de la participación que tiene el Partido Popular sobre la formación y creación de opiniones y actitudes negativas hacia la inmigración en la sociedad española, necesitábamos unos discursos públicos que fueran fácilmente accesibles para el mayor número posible de audiencia. Parece evidente, que tanto los debates parlamentarios como las entrevistas políticas, debido a su gran cobertura mediática, llegan a una gran cantidad de gente. Por esta razón, se descartaron otros géneros políticos de menor difusión.

\subsection{Métodos de análisis}

Para presentar el análisis de forma que sea más accesible para el lector, haremos una enumeración de los distintos temas que aparecen recurrentemente en el discurso del Partido Popular sobre inmigración. El tema es el asunto o la materia principal sobre lo que trata el discurso. Se trata de una entidad abstracta y generalizada que suele tener una estructura nominativa. No debemos confundir el tema con la macroproposición, que se presentan con estructura oracional y son las realizaciones y articulaciones particulares de dichos temas.

Consecuentemente, dentro de cada tema, realizaremos un análisis sistemático de las macroproposicones concretas que lo configuran. Dentro de estas macroproposiciones, atenderemos a la representación de los distintos actores y sus roles, así como las relaciones entre ellos, los atributos y los actos que se asignan a cada uno de ellos. Cuando sea pertinente, analizaremos también la modalidad de dichas macroproposiciones.

Así, distinguiremos entre modalidad epistémica (la expresión del grado de certeza o duda que el emisor muestra con respecto a la verdad de la macroproposición), modalidad deóntica (que indica el grado de obligatoriedad de que se produzca el contenido enunciado) y la modalidad axiológica (que muestra la actitud del emisor sobre lo enunciado) (Bybee y Fleischman, 1995). Igualmente, para tener una idea más concreta sobre la superestructura y la coherencia interna del discurso, analizaremos la relación secuencial de las distintas macroproposiciones encontradas.

Para obtener un análisis más inteligible, que permita comprender en profundidad los temas empleados más frecuentemente por el Partido Popular en su discurso sobre inmigración, se han contado el número de veces que aparece cada uno de los temas en el corpus seleccionado y se ha establecido una proporción entre todos ellos para entender, en forma de porcentaje, a qué temas se recurre con más o menos frecuencia. Así pues, después de la enunciación de cada tema aparecerá, entre paréntesis, el porcentaje que indicará la frecuencia con que este tema aparece. 
Igualmente, se ha contado la frecuencia con la que aparecen las distintas macroproposiciones (que también aparecerá entre paréntesis tras la enunciación de cada una de ellas). Por tanto, se ha resumido cada párrafo (o a veces varios párrafos) con una macroproposición (MP) y, seguidamente, se han contado las veces que aparece cada macroproposición a lo largo de todo el corpus. De este modo, se ha observado que, manifestaciones similares de las mismas macroproposiciones, se repetían de forma constante. Estas manifestaciones se han agrupado dando lugar a macroproposiciones generalizadas.

Finalmente, cabe destacar que, se han contado no solo los temas y las macroproposicones que aparecen de forma explícita en el texto, sino también se han contado las veces en las que, el significado de las mismas, puede ser inferido del discurso, sin que necesariamente aparezca una referencia explícita en el mismo. Por tanto, se ha realizado un análisis no solo de contenido, sino también de significado, teniendo en cuenta igualmente el cotexto y el contexto, que permiten inferir la formulación de cada tema y macroproposición.

\section{El análisis}

Para comenzar, señalaremos que, a lo largo del corpus analizado, se encuentran los siguientes ocho temas, de forma recurrente y constante:

Tabla 1. Temas en la representación social de la inmigración en el discurso del Partido Popular

\begin{tabular}{|l|l|}
\hline Temática & Frecuencia \\
\hline Defensa de la nueva ley & $22,11 \%$ \\
\hline La delincuencia & $13,68 \%$ \\
\hline La llegada de inmigrantes & $12,63 \%$ \\
\hline La exaltación de España & $11,58 \%$ \\
\hline Los derechos y las obligaciones & $11,41 \%$ \\
\hline Racismo y Xenofobia & $10,88 \%$ \\
\hline La cultura & $9,82 \%$ \\
\hline La religión & $4,56 \%$ \\
\hline
\end{tabular}

Como vemos, la temática más recurrente, con diferencia, es la defensa de la nueva ley de extranjería, puesto que, como ya hemos señalado, esta ley recibió numerosas críticas por parte de los otros partidos políticos. Con el fin de que el lector tenga siempre presente aquellos temas sobre los que se reincide más, en 
nuestro análisis mantendremos este orden de mayor a menor frecuencia. A continuación, realizaremos un estudio detallado de cada una de las temáticas y, en particular, de las macroproposiciones que las componen, así como de las relaciones entre ellas.

1. Defensa de la nueva Ley de Extranjería LO 8/2000 (22,11\%)

Esta defensa se articula en torno a tres macroproposiciones generales:

a) Hay que luchar contra las mafias (52,38\%); observemos el siguiente ejemplo:

(1) El Gobierno lucha seriamente contra la inmigración ilegal, que tampoco debemos olvidar que está promovida por mafias que se aprovechan de estas personas, explotándolas y poniendo en la mayoría de las ocasiones en riesgo sus propias vidas, como hemos podido ver casi todos los días desgraciadamente a través de los medios de comunicación. (Antonio González. Congreso. 15-10-03)

Como puede observarse, se establece que para evitar que existan las mafias que trafican con personas, hay que luchar contra la inmigración ilegal, ya que esta está siempre promovida por las mafias. Y puesto que la nueva ley tiene como punto fundamental la lucha contra la inmigración de carácter ilegal, esta lucha contra las mafias sirve como justificación del control de este tipo de inmigración.

b Hay que evitar «el efecto llamada» $(15,87 \%)$; esta segunda macroproposición sirve de justificación para la defensa de la nueva ley, ya que ésta se establece como un mecanismo necesario para evitar «el efecto llamada», es decir que vengan más inmigrantes a España, como sucede en el siguiente fragmento:

(2) La nueva ley, el final del proceso de regularización y los convenios que firmaremos con los países de origen de estas personas frenarán el llamado «efecto péndulo» que se está produciendo ahora. Y lo espero fundamentalmente por los propios inmigrantes, porque son personas que se están jugando la vida por venir aquí, donde hasta ahora tenían la garantía de que iban a ser explotados. Un Estado democrático como el nuestro no puede mirar a otro lado cuando sabe que se está explotando a estas personas. (Entrevista a Enrique Fernández-Miranda. 17-12-00)

Así, que vengan más personas migradas se presupone negativo (Simon-Vandenbergen, et al., 2007). Se observa, por tanto, la representación de la inmigración como una amenaza, como un mal a evitar que repercute negativamente tanto a las personas migradas como a la sociedad española y, 
por ello, se hace necesario tener unas leyes restrictivas que impidan que más gente quiera venir a España. De este modo, el PP se representa positivamente como un partido preocupado por el bienestar de las personas migradas.

Las personas migradas, en este caso, aparecen suprimidas e impersonalizadas (Van Leeuwen, 1995, 1996), ya que, aunque se infiere que el efecto llamada se refiere a que vengan más personas migradas, estas personas no aparecen referidas en ningún momento. Así, esta abstracción de la necesidad de evitar un efecto sirve como atenuador de lo potencialmente negativo que sería expresar directamente que hay que evitar que vengan más personas migradas a España.

c) Hay que distinguir entre inmigrantes legales e ilegales $(31,75 \%)$; esta tercera macroproposición obedece a un objetivo de legitimación de una de las secciones más criticadas de la ley, que es la distinción entre inmigrantes regularizados y no regularizados. Para justificar este apartado de la ley, se alega que esta distinción es en beneficio de los inmigrantes (regularizados, se entiende) para poder proteger sus derechos y garantizar su integración, como vemos:

(3) La principal reforma que establece la ley es la distinción entre legales e ilegales, eso lo entiende todo el mundo, y solamente la inmigración legal garantiza de verdad la integración del emigrante. (Entrevista a Mariano Rajoy. 9-7-00)

Nos encontramos ante la interposición de la legalidad como el único camino para alcanzar la integración del colectivo inmigrante, aunque esta relación directa que se establece no es, en ningún momento, explicada ni especificada. Esta generalización un tanto sesgada, se extiende hacia su contrario, es decir: la inmigración ilegal favorece la explotación, la no integración y la acción de las mafias.

En las tres macroproposiciones, se presupone, por tanto, que todo inmigrante no regularizado está siendo explotado, es incapaz de integrarse y ha venido a España engañado por las mafias. La realidad, en cambio, es que, la mayoría de las personas migradas que permanecen en España de manera ilegal han entrado al país a través de los aeropuertos, como turistas. ${ }^{1}$ Sin embargo, la interposición

1. Numerosos periódicos (http://www.abc.es/hemeroteca/historico-23-09-2006/abc/Madrid/unos-1500inmigrantes-ilegales-entran-cada-dia-por-barajas-como-turistas_1423433342392.html; http://www.20minutos.es/noticia/155270/0/inmigrantes/ilegales/barajas/; http://www.elpais.com/articulo/espana/millar/inmigrantes/bolivianos/entran/diariamente/aeropuerto/Bar ajas/elpepuint/20070104elpepinac_16/Tes) han puesto de manifiesto que, según el Sindicato Unificado de Policía, más del 55\% de los inmigrantes, que posteriormente permanecen en España de manera ilegal, llegan a España a través de los aeropuertos como turistas. 
de este conocimiento de grupo como si fuera un conocimiento comúnmente compartido, conlleva la representación negativa de las personas migradas, que se presentan como víctimas explotadas y engañadas.

Así, mientras, a) y $c$ ) establecen la figura del inmigrante como un sujeto pasivo, que es víctima de las mafias y de la explotación, en $b$ ) sencillamente las personas migradas son suprimidas e impersonalizadas. El PP, en cambio, en las tres macroproposiciones se interpone como un agente activo, que tiene que proteger a los inmigrantes.

Finalmente, las diversas manifestaciones de cada una de estas tres macroproposiciones, se enuncian a través de una modalidad deóntica que expresa una obligación y una certeza sobre la necesidad de luchar contra las mafias, evitar el efecto llamada y distinguir entre inmigrantes en situación legal o ilegal, de manera que la ley se interpone como la herramienta necesaria para proteger a las personas migradas.

\section{La delincuencia $(13,68 \%)$}

En el discurso del Partido Popular sobre inmigración es una constante la asociación del aumento de la delincuencia con el incremento de la inmigración. En concreto, esta temática se materializa en dos macroproposiciones generales:

a) La inmigración va ligada al aumento de la delincuencia $(68,42 \%)$; se proclama una relación directa entre la inmigración y la delincuencia, de manera que a las personas migradas se les atribuye un rol activo en el incremento de la misma. Esta atribución de actos provoca que se perciba a las personas migradas como a un colectivo social (delincuente), y no como a individuos, con rasgos o características particulares, como vemos:

(4) La inmigración ilegal va ligada al aumento de delincuencia. Una prueba: el $90 \%$ de los detenidos que ingresan en la Modelo son inmigrados. (Entrevista a Alberto Fernández. 15-9-02)

De este modo, se crean estereotipos de carácter negativo sobre este grupo social que, innegablemente, condicionarán las interrelaciones del endogrupo con los miembros del exogrupo.

b) Los datos dicen que muchos delitos son cometidos por inmigrantes $(31,58 \%)$; hay una tendencia general a establecer esta relación entre inmigración y delincuencia acompañada de afirmaciones categóricas que la representan 
como un hecho objetivo, incontestable, evidente, e irrefutable. Estas afirmaciones categóricas intensifican el significado dotándolo de una aparente objetividad, que se ve reforzada por la inclusión de datos numéricos que permiten, de algún modo, delegar la responsabilidad de lo dicho: como si estas afirmaciones no provinieran del Partido Popular, sino de algún tipo de organismo, que maneja estos datos y el Partido Popular fuera un mero trasmisor de los mismos, como puede observarse:

(5) - ¿Hay un vínculo serio entre inmigración y delincuencia?

- Los datos son incontestables. Ha habido un crecimiento de la inseguridad y de la delincuencia que en gran medida va ligada a la inmigración ilegal. Pero no tiene sentido establecer un vínculo directo entre inmigración y delincuencia. (Entrevista a Josep Piqué. 21-6-02)

Así pues, mediante la referencia a unos datos (cuya proveniencia rara vez se menciona) que «hablan», «dicen», «reflejan» o «ponen de manifiesto» que numerosos delitos son cometidos por inmigrantes, se pretende camuflar la autoría de estas afirmaciones negativas, personificando y convirtiendo a «los datos» en el agente que expresa dichas afirmaciones. Esto, innegablemente, contribuye a mitigar presentación negativa del Partido Popular ya que, al presentarse como meros trasmisores, minimizan la posible imagen negativa (Brown y Levinson, 1987) que del partido se proyectaría al señalar directa y explícitamente que los inmigrantes son delincuentes.

Entre estas dos macroproposiciones encontramos una relación secuencial funcional (Van Dijk, 1977, 1984). Las relaciones secuenciales funcionales son aquellas en las que una proposición se define con respecto a la otra. Ejemplos de este tipo de relaciones son las ilustraciones, las explicaciones o los ejemplos. En el caso que nos ocupa, la segunda macroproposición es una especificación, o incluso una ilustración de la primera. Si bien, como hemos visto, la segunda macroproposición tiene la función de delegar la responsabilidad del PP sobre lo dicho, a su vez sirve de refuerzo de la primera, puesto que a través de unos datos numéricos concretos, se intenta dotar de cierta objetividad y rigor a lo dicho en la macroproposición anterior.

Asimismo, encontramos la asignación de un rol activo a las personas migradas como agresores que suponen una amenaza para la seguridad ciudadana de España, que aparece como víctima pasiva. El PP se representa como el defensor de la sociedad española frente a la amenaza latente. 
3. La llegada ilegal de inmigrantes $(12,63 \%)$

La llegada ilegal de inmigrantes a España es otra temática recurrente que se articula en torno a dos macroproposiciones fundamentales:

a) Un número muy elevado y constantemente creciente de inmigrantes llega a España (46,30\%); hay una tendencia generalizada a mencionar las continuas llegadas de inmigrantes, que se conceptualiza como una invasión, mediante el uso de expresiones hiperbólicas y metáforas asociadas a desastres naturales, guerras y otras conceptualizaciones negativas. Esto tiene, inexorablemente, connotaciones que alimentan la percepción social negativa del fenómeno y facilitan su representación como una amenaza que, a su vez, obliga a adoptar una actitud defensiva frente a la misma, como se observa:

(6) Lo que hay es una preocupación respecto a oleadas de inmigración ilegal que vienen de determinados países, y la mayoría no son precisamente latinoamericanos, sino del Magreb, del África subsahariana y del propio continente asiático. (Entrevista a Josep Piqué. 19-6-02)

Así, rara vez se hace referencia al número de inmigrantes que llegan, sino que se utilizan sustantivos como oleada, avalancha o aluvión para conceptualizar e hiperbolizar el fenómeno de manera negativa.

b) Los inmigrantes colapsan nuestro sistema (53,70\%); cuando se habla de estas avalanchas es frecuente hacer mención al colapso o el desbordamiento que esto nos supone a nosotros: ya sea por el desbordamiento de los sistemas de ayuda humanitaria, que se ven incapaces de asistir a tantas personas en las costas, o ya sea porque nuestra sociedad se ve incapaz de acoger y gestionar a tanta gente:

(7) Por ello, tenemos que ser capaces de acoger dignamente a los que quieren venir, pero sin poner en peligro nuestra capacidad de respuesta a los españoles, a los que tenemos absoluta obligación de atender con todas las garantías. La llegada desordenada de inmigrantes no puede repercutir las ayudas a nuestros ciudadanos. (Entrevista a Ignacio González. 23-9-02)

Como vemos, encontraremos continuas alusiones a nuestra incapacidad para dar trabajo a todos, o nuestra incapacidad para asegurar servicios sociales para todos (como la sanidad, la educación, etc.). Vemos aquí que esta es, probablemente, la única representación negativa del endogrupo, que se representa como «incapaz». Si bien, vemos que esta autopresentación negativa sirve como argumentación para poner freno a la llegada de inmigrantes y para 
representarlos, una vez más, como una amenaza para nuestro estado de bienestar.

La relación secuencial entre estas dos macroproposiciones es de carácter condicional. Las relaciones secuenciales condicionales son aquellas que se interrelacionan a través de una causa, una razón o una consecuencia. En este caso, la segunda macroproposición se interpone como una consecuencia de la primera. Y, por tanto, una vez más, la conclusión implícita que se impone es que, para evitar que los inmigrantes colapsen nuestro sistema, es necesario limitar su llegada a nuestro país. Así, la inmigración se representa como una amenaza para nuestro estado de bienestar y nuestro rechazo a la llegada de más personas migradas se justifica mediante la presentación de este rechazo como forma de defensa ante dicha amenaza.

\section{La exaltación de España $(11,58 \%)$}

Asistimos a la exaltación de España, que se presenta como un país hospitalario, habituado a la diversidad, de talante abierto, tolerante, sin partidos de extrema derecha, etc. Esta exaltación, en el discurso del Partido Popular, toma forma a partir de tres macropoposiciones, cada una de ellas con una función específica:

a) España quiere ofrecer puestos de trabajo a todos, pero nuestra capacidad de acogida es limitada (27,27\%); las macroproposiciones que componen esta macroproposición general cuentan con una estructura idéntica, en la que la primera parte antes del conector adversativo (pero, sin embargo, no obstante, etc.) funciona como preámbulo antes de introducir una idea negativa:

(8) España quiere seguir ofreciendo puestos de trabajo mientras siga teniendo puestos de trabajo que ofrecer y, de momento, vamos a poder hacerlo en una temporada. Pero la realidad es que nuestra capacidad de acogida está limitada y la exigencia de que vengan y permanezcan entre nosotros de manera legal, creo que es una exigencia razonable. (Entrevista a Enrique Fernández-Miranda. 23-1-01)

De esta forma, en esta primera parte se introduce una autopresentación positiva del PP o de España, que acompaña al mensaje principal de carácter negativo, que es que España tiene una capacidad de acogida limitada. Este movimiento se denomina disclaimer (Van Dijk, 2003 e) y sirve para mitigar (a la vez que, de algún modo, legitimar) el hecho de que no podemos acoger a personas de forma ilimitada. De este modo, nuestra incapacidad se mitiga con nuestra buena voluntad. 
En la segunda parte del disclaimer, en cambio, las personas migradas aparecen excluidas, de forma que se representan en un segundo plano mediante la abstracción de la capacidad de acogida. Obviamente, esta capacidad de acogida se refiere a la acogida de las personas migradas, pero estas no aparecen referidas en ningún momento, si bien su referencia puede inferirse. Esta no referencia explícita de las personas migradas, tiene un efecto deshumanizador que sirve igualmente como estrategia de mitigación de la incapacidad de acogida del endogrupo.

b) España es un país hospitalario y a cambio solo pedimos... (42,42\%); esta segunda macroproposición, aunque también tiene una función mitigadora, se presta para la exigencia de reciprocidad, como vemos:

(9) España siempre ha sido hospitalaria, pero nuestra tolerancia debe ser correspondida con la suya. Por ejemplo, me preocupa mucho que algunas familias magrebíes saquen a sus hijas de la escuela a los 12 años, porque así pierden la igualdad de oportunidades respecto a los niños. (Entrevista a Enrique Fernández-Miranda. 25-6-00)

España se representa como un país hospitalario y acogedor y se exige a las personas migradas que realicen algo a cambio, o procedan de una determinada manera (que trabajen, que permanezcan en España de forma legal, que respeten y se adapten a nuestra constitución, nuestra cultura, nuestra religión, etc.). A las personas migradas se las representa pasivamente como beneficiarios de nuestra hospitalidad y se interpone a España como elemento necesario para su activación, de modo que las personas migradas participen activamente en la sociedad, trabajando, adaptándose, integrándose, etc. Consecuentemente, una vez más las personas migradas aparecen representadas como incapaces de ejercer sus obligaciones por sí mismas y, por tanto, se hace necesario que España les recuerde estas obligaciones.

c) España es una sociedad abierta y tolerante (39,30\%); Esta tercera macroproposición cumple la función de exaltar España, como forma de autopresentación positiva, sin más objetivo que enfatizar los aspectos positivos del endogrupo, como puede observarse:

(10) Valores como los de la tolerancia y el pluralismo cultural y étnico, son los que hay que inculcar a los niños y recordar a los adultos. De ese modo, preservaremos una sociedad como la española abierta, hospitalaria, habituada a la diversidad... España puede presumir de no tener partidos de extrema derecha y ese es un gran mérito de nuestra sociedad. Un mérito del que carecen otros países de nuestro entorno. (Entrevista a Enrique Fernández-Miranda. 4-11-01) 
Así, por tanto, las tres macroproposiciones que encontramos en el discurso del PP sobre la exaltación de España, responden a un intento de autopresentación positiva; no obstante, cada una de ellas tiene una función particular que suele estar ligada a estrategias de mitigación y legitimación de nuestros aspectos negativos. De este modo, se proyecta una imagen positiva de España y del Partido Popular, que se contrapone a la proyección de la imagen negativa de las personas migradas.

5. Los derechos y las obligaciones $(11,41 \%)$

Este tema gira en torno a dos macroproposiciones básicas:

a) Los inmigrantes tienen derechos y obligaciones (65\%); observamos que mientras las obligaciones que se adjudican a las personas migradas aparecen detalladas y especificadas, los derechos aparecen meramente referidos y rara vez (solo dos veces a lo largo de todo el corpus) explicitados o explicados. Por tanto, la mención a sus derechos parece obedecer a una estrategia de mitigación, para minimizar el efecto de la imposición por nuestra parte de sus obligaciones (Brown y Levinson, 1987), como vemos:

(11) Los ciudadanos extranjeros que vienen a España tienen que saber que aquí hay unas reglas de juego que son las que se dan los españoles y que responden a unos valores, a unos principios y a una historia que son los que están en la Constitución. Y si aquí la enseñanza es obligatoria hasta los 16 años, es obligatoria para un ciudadano español y para cualquier ciudadano que viene aquí, que además de tener derechos, tiene que tener obligaciones, y malo es que los representantes políticos no digamos esto, no vaya a ser que nos lo digan otros. (Mariano Rajoy. Congreso. 8-5-02)

La modalidad deóntica con que se expresan estas proposiciones nos sugiere que, se considera un derecho y una obligación propia de España el exigir a los inmigrantes que acepten y respeten nuestra constitución, nuestras normas de convivencia, nuestras reglas del juego, nuestras costumbres, nuestra ley, nuestra democracia, nuestra cultura, nuestra religión, etc. Sin embargo, en ningún momento se habla de la necesidad de la existencia de una reciprocidad, ni de nuestra obligación de respetar y aceptar sus culturas, sus religiones, sus costumbres etc. De este modo, se prioriza a los ciudadanos autóctonos y se les sitúa jerárquicamente por encima de las personas migradas, a las que se presupone supeditadas a los autóctonos. 
b) Debemos controlar la llegada de inmigrantes para poder garantizar sus derechos fundamentales (35\%); el poder garantizar unos derechos fundamentales (que, una vez más, no aparecen especificados) a los inmigrantes, sirve como justificación para poner freno a la llegada de los mismos, puesto que se establece que, si llegan más inmigrantes de los que podemos acoger, el gobierno no será capaz de garantizarles una serie de derechos fundamentales:

(12) Lo que me preocupa es que nosotros seamos capaces de acoger a personas que puedan llevar una vida digna [...] Porque si al final entran personas en número ilimitado, lo que sucederá es que no podremos garantizarles nada. (Entrevista a Mariano Rajoy. 9-7-00)

Como vemos, los inmigrantes que llegan a España de manera ilegal aparecen representados como agresores activos que suponen una amenaza para sí mismos (porque llegan descontroladamente) y para nuestro estado de bienestar (porque colapsan las organizaciones que lo garantizan). Por el contrario, el PP se autorepresenta como el bienhechor que se preocupa por salvaguardar los derechos fundamentales de los inmigrantes. Asimismo, encontramos de nuevo una modalidad deóntica que expresa la obligatoriedad de controlar la llegada de inmigrantes para poder salvaguardar sus derechos fundamentales. Se dictamina, pues, como hecho incuestionable que solo a través del control de las fronteras se puede garantizar el bienestar de los inmigrantes.

Encontramos un silogismo entre el contenido expresado en la primera macroproposición y el de la segunda: la primera premisa es que los inmigrantes tienen derechos, la segunda premisa es que no podemos garantizar los derechos a todos. En consecuencia, la conclusión implícita que se impone es que tenemos que controlar la llegada de inmigrantes para garantizar sus derechos fundamentales. Así, por tanto, a través de estas dos macroproposiciones, se produce una coherencia interna en la argumentación para la justificación del control de la inmigración.

\section{Racismo y xenofobia $(10,88 \%)$}

En esta temática encontramos dos macroproposiciones fundamentales:

a) España no es un país racista (48,39\%); asistimos a una negación de la existencia de racismo en España. O bien se mitiga, diciendo que es un hecho muy puntual, que no se puede considerar como un problema; o bien se niega su 
existencia, alegando que no es racismo, sino miedo o preocupación lo que la gente siente. Esta mitigación o negación de la existencia de actitudes racistas comporta la representación positiva de los españoles como una sociedad tolerante y hospitalaria, como vemos:

(13) Quisiera hacer algunas consideraciones iniciales. Primero, si nos comparamos, que parece la comparación lógica, con los países de nuestro entorno, los de la Unión Europea, España no es un país -y ojalá no lo sea en el futuro-donde el racismo y la xenofobia sean un problema real. Esa es mi opinión. No son los españoles racistas, en términos generales. Mariano Rajoy (PP). Congreso. 8-5-03)

De este modo, no solo se consigue alabar a la posible audiencia (es decir, a los votantes españoles), sino que, implícitamente, también se está ensalzando la labor del gobierno como gestor de una política de inmigración adecuada, que permite e impulsa la integración de los inmigrantes y repele y rechaza toda actitud racista. Este no reconocimiento de la existencia de actitudes racistas en España acarrea una consecuencia inquietante, que ya señaló Torrens (2002) en su tesis doctoral sobre el racismo cultural. Esto es, como el problema no existe, no hay que implantar ningún tipo de medidas para solucionar los (pocos o muchos) problemas de convivencia que se daban entonces y se siguen dando ahora en nuestra sociedad a causa de actitudes racistas e intolerantes.

b) La inmigración ilegal puede generar racismo $(51,61 \%)$; la posibilidad de que se engendre un racismo, una marginalidad o una exclusión social, que actualmente no existen en la sociedad, se establece como argumento para justificar determinadas decisiones políticas relacionadas con las partes más restrictivas de la ley de extranjería.

Por tanto, se implica que dichas políticas son buenas para los inmigrantes porque, gracias a ellas, no tendrán que padecer actitudes racistas, ni marginalidad, ya que se establece una relación directa entre legalidad e integración y entre ilegalidad y marginalidad. Sin embargo, no se explicita de ningún modo, la relación entre la ilegalidad, la marginalidad y el racismo, ni cómo el hecho de ser un inmigrante, que está de forma ilegal en España, puede hacer nacer actitudes racistas en una sociedad, que ha sido calificada como tolerante, hospitalaria, etc. Si esto fuera cierto, de esta relación causal, se derivaría que, en cambio, un inmigrante que está de forma legal en España, no hace despertar actitudes racistas: como si el nacimiento y la perpetuación de actitudes racistas estuviera determinada por la tenencia o carencia de papeles en regla por parte de los inmigrantes: 
(14) La eliminación de las fronteras es una solución utópica, radicalizada, irresponsable y que al final produciría brotes de racismo, xenofobia y el desarrollo de políticas de extrema derecha contra la tolerancia, la interculturalidad y los inmigrantes. (Entrevista a Antonio Lis (PP). 10-2-02)

Igualmente, con este razonamiento que se establece como hecho incuestionable, se produce una victimización de la sociedad española y una criminalización del inmigrante que está en España de forma ilegal, ya que se dibuja a la sociedad española como víctima del inmigrante: como si el hecho de que haya inmigrantes de manera ilegal en España, nos avocara indefectiblemente a la formación de actitudes racistas (Ryan, 1976; Janoff-Bulman, Timko y Carli, 1985).

Si prestamos atención a la estructura de la macroproposición, veremos que el sujeto es «la inmigración ilegal». Por tanto, es la inmigración la causa de los posibles brotes de racismo. De este modo, la responsabilidad recae sobre la inmigración y no sobre nosotros, los españoles. Consecuentemente, la conclusión que se impone es que hay que evitar la llegada descontrolada de estos inmigrantes a España, para prevenir dichas actitudes racistas, que nacerían inevitablemente en nosotros.

Como vemos, entre estas dos macroproposiciones, existe una secuencia condicional, ya que se establece una relación consecuencial entre una y otra. La conclusión implícita que se transmite es que en España no hay racismo, siempre y cuando no haya demasiada inmigración ilegal, porque esta puede generar racismo y, por tanto, hay que controlar y limitar el número de inmigrantes que llegan a España de forma ilegal. Esta secuencia de macroproposiciones, por tanto, parece obedecer a esquemas cognitivos subyacentes grupales, que presentan positivamente al endogrupo (como tolerante, no racista, etc.) y negativamente al exogrupo (como una amenaza para nuestra tolerancia).

\section{La cultura $(9,82 \%)$}

El discurso sobre la cultura se manifiesta a partir de dos macroproposiciones:

a) Ellos tienen una cultura diferente $(71,43 \%)$; se tiende a destacar y enfatizar los aspectos negativos de su cultura supeditándola, de algún modo, a la nuestra que, por contraposición, aparece representada como superior En líneas generales, hay una inclinación a representar la cultura del colectivo inmigrante como una amenaza, que vulnera los principios del Estado democrático y como una cultura ostensiblemente machista y fundamentalista, que 
atenta contra nuestra Constitución y nuestro Estado de bienestar. Una vez más, asistimos a la proyección de prejuicios negativos sobre este colectivo, a partir de generalizaciones que engloban a todo el colectivo, categorizándolo de manera homogénea: como si todos los inmigrantes tuvieran la misma cultura, que a su vez es completamente distinta a la nuestra.

En general, dentro del discurso sobre la cultura, se tiende a simpatizar más con los países de Latinoamérica, que se representan como inmigrantes más cercanos a nuestra cultura y más fácilmente integrables. Esto se contrapone a los inmigrantes procedentes de países islámicos, que se representan como una amenaza por tener una cultura irreconciliable con la nuestra y abiertamente hostil e impositiva.

b) Ellos deben adaptarse (28,57\%); la línea argumentativa del discurso del Partido Popular sobre la cultura presupone la existencia de una intolerancia por parte de los inmigrantes hacia nuestra cultura. Como consecuencia, se establece como necesidad el hecho de que los inmigrantes tienen que hacer lo posible por adaptarse e integrarse en nuestra cultura.

Así pues, por un lado, a las personas migradas se les asigna un rol activo impositivo y se les categoriza de manera homogénea y generalizada como intolerantes e intransigentes. Por otro lado, se tiende a degradar $s u$ cultura y sus costumbres, enfatizando sus aspectos más negativos, como vemos:

(15) - España siempre ha sido hospitalaria, pero nuestra tolerancia debe ser correspondida con la suya. Por ejemplo, me preocupa mucho que algunas familias magrebíes saquen a sus hijas de la escuela a los 12 años, porque así pierden la igualdad de oportunidades respecto a los niños.

- ¿Por qué lo hacen?

- Consideran que deben tener una educación distinta y aprender lo necesario para estar en casa. Ellos deben adaptarse a nosotros. (Entrevista a Enrique FernándezMiranda (PP). 25-6-00)

\section{La religión $(4,56 \%)$}

Como vemos en el fragmento (15) el tema de la religión a menudo aparece solapado con el tema de la cultura. Igualmente, este tema se articula en torno a dos macroproposiciones:

a) Su religión favorece la desigualdad entre hombre y mujer (43,85\%); aparecen numerosas referencias a la religión de ellos, a través de la crítica (explícita o implícita) al uso del velo en las mujeres, que aparece siempre 
representado como un símbolo de la desigualdad de géneros y como una imposición hacia la mujer.

Esta representación negativa de la religión de ellos, como favorecedora de la desigualdad, sumado a la presuposición de que ellos son anticonstitucionales e intolerantes, es coherente con la macroestrategia general de enfatizar, exagerar, e incluso malinterpretar los aspectos negativos del exogrupo. A su vez, esta representación negativa, por contraposición, fomenta la autopresentación positiva del endogrupo como país favorecedor de la igualdad, la tolerancia y lo constitucional. Por tanto, se presupone que en nuestra sociedad sí que existe una igualdad entre hombre y mujer. Asimismo, esta polarización contribuye a la visión del exogrupo como una amenaza que vulnera los principios del estado democrático y, por tanto, se proyecta la necesidad de proteger nuestra identidad, nuestra cultura y, en general, nuestra sociedad frente a dicha amenaza.

b) Ellos deben adaptarse (56,15\%); al igual que sucede con el discurso sobre la cultura se establece que los inmigrantes tienen la obligación de adaptarse a la religión cristiana, ya que la musulmana fomenta la desigualdad entre hombre y mujer y, por tanto, se considera peor que la nuestra. Una vez más, se tiende a englobar a todo el colectivo inmigrante dentro de una misma religión (la musulmana) dotándolo, además, de un fundamentalismo y una radicalización generalizada. Esta visión homogeneizada y generalizada que se proyecta sobre los inmigrantes contribuye indudablemente a la creación de prejuicios negativos hacia este colectivo.

Finalmente, cabe destacar que el discurso del PP sobre la cultura y la religión de los inmigrantes tiene una estructura macroproposicional secuencial muy marcada. En ambos casos y, como norma general, encontramos una relación contrastiva entre la presentación negativa de $s u$ cultura y su religión, que se contrapone con la presentación positiva de nuestra cultura y nuestra religión. la conclusión obvia que se deriva de este razonamiento, es que deben ser ellos los que se adapten a nosotros. En todos los casos contemplados sobre la cultura y la religión, se observa una modalidad deóntica que expresa la obligación incuestionable de que sean ellos los que tienen que adaptarse a nosotros.

\section{Conclusiones}

A la luz de los resultados obtenidos, podemos concluir que la representación social sobre las personas migradas que se desprende del discurso del Partido Popular, es en términos generales negativa. Hemos visto que, por un lado, se 
tiende a conceptualizar la llegada ilegal de inmigrantes a España como una amenaza para la sociedad española y para las propias personas migradas.

La amenaza para la sociedad española se presenta a través de la proyección de una serie de prejuicios sobre la naturaleza de las personas migradas. Asistimos a una generalización que tiende a agrupar a todo el colectivo inmigrante de manera homogénea. Obviamente, esto provoca que los atributos y los actos que se les asigna son aplicados a todos los miembros de este colectivo, que serán percibidos como un grupo y no como individuos.

Así, encontramos que, en primer lugar, se asocia a las personas migradas con el incremento de la delincuencia en España. En segundo lugar, a estas personas se les presupone intolerantes, anticonstitucionales, antidemocráticas, impositivas e intransigentes. Consecuentemente, se les presupone una predeterminación a intentar cambiar nuestras costumbres, nuestras leyes, nuestra cultura, nuestra religión, etc. Asimismo, se tiende a enfatizar (y exagerar) los aspectos negativos de su cultura y su religión, de manera que estas se presenten negativamente en contraposición con las nuestras. En tercer lugar, se enfatiza, implícita y explícitamente, que la inmigración colapsa y desborda nuestra capacidad de acogida, que es limitada. En respuesta a esto, se sitúa a la sociedad española, que se representa como víctima, en una posición defensiva, ante la amenaza patente.

La amenaza para las propias personas migradas se consigue conceptualizar a partir de asociaciones que vinculan a (toda) la inmigración de carácter ilegal con las mafias que trafican con personas y con la explotación. De este modo, se justifica la necesidad de controlar la llegada de personas migradas en su propio beneficio, para impedir que estas personas sufran.

Por otro lado, esta representación negativa de la inmigración se contrapone con la representación positiva del Partido Popular y de España, que se establece como un país hospitalario, tolerante, de talante abierto, donde no existen actitudes racistas ni xenófobas importantes. Sin embargo, se proclama que existe la posibilidad de que surjan brotes racistas, si España acoge a demasiadas personas migradas. Así, observamos de nuevo, que se presenta a la inmigración como un peligro para el bienestar de la sociedad española. Como consecuencia, obtenemos una representación de las personas migradas sesgada, que tiende a presentarlas en términos generalizadores, categóricos y negativos.

Así, por tanto, encontramos cómo la(s) ideología(s) del Partido Popular determinan esta forma de representación negativa del exogrupo, en contraposición con la representación positiva del endogrupo. Para poder enunciar la lista de las ideologías que gobiernan el discurso del Partido Popular, sería necesario realizar un análisis más local de su discurso. Pero sí que podemos hablar de actitudes negativas hacia la inmigración, que se proyectan en su discurso a nivel global. 
Igualmente y, para finalizar, cabe destacar que, esta representación negativa de la inmigración en el discurso del PP, es coherente con los resultados obtenidos en una investigación más amplia, ${ }^{2}$ que se completa con el microanálisis léxico-semántico del corpus seleccionado y las estrategias retórico-argumentativas empleadas por el Partido Popular en su discurso sobre inmigración.

\section{Bibliografía}

Allport, G. W. (1977): La naturaleza del prejuicio, Buenos Aires, Editorial universitaria de Buenos Aires.

BAddeley, A. (1999): Memoria humana. Teoría y Práctica, Madrid, McGraw Hill.

Bañón, A. M. (2002): Discurso e inmigración. Propuestas para el análisis de un debate social, Murcia, Universidad de Murcia.

Brown, P.; S. C. Levinson (1987[1978]): Politeness. Some universals in language usage, Cambridge, Cambridge University Press.

Brown, R. (1988): «Intergroup relations» en Hewstone, M. et al (eds.) (1988): Introduction to social Psychology. A European perspective, Oxford, Basil Blackwell. 479-515.

Bybee, J.; S. Fleischman (1995): Modality in Grammar and Discourse, Amsterdam/Philadelphia. John Benjamins Publishing Company.

Chilton, P.; C. Schäffner (1997): «Discourse and politics», en VAn DiJK, T. A. (ed.) (997): Discourse studies: A multidisciplinary introduction, London, Sage. 206-230.

Dios, S. (2005): «Inmigración y extranjería en el debate parlamentario español» en A. Pedreño, M; M. Hernández (coords.): La condición inmigrante. Exploraciones e investigaciones desde la región de Murcia, Murcia, Universidad de Murcia. 175-180.

García O’ Meany, M. (2002): Yo no soy racista pero- - justificando la discriminación, Barcelona, Intermón Oxfam.

Graciano, P. (2006): De Aznar a Rajoy, 1990-2007: la maldición de Casandra: los secretos de la derecha española, Tres Cantos, Foca.

Hamilton, D. L.; T. Trolier (1986): «Stereotypes and Stereotyping, an overview of the cognitive approach» en Dovidio, J. F.; S. L. GAERTnER (eds.) (1986): Prejudice, Discrimination and Racism, San Diego, Academic Press. 127-157.

IZQUIERDO, A. (2002): «La inmigración en España entre dos regularizaciones», Economistas, 20(91): 277-282.

2. Este artículo se deriva de la investigación llevada a cabo para la confección de una tesis doctoral en la Universitat Pompeu Fabra sobre el discurso político del Partido Popular sobre inmigración. 
Janoff-Bulman, R.; C.Timko C; Ll. Carli (1985): «Cognitive biases in blaming the victim», Journal of Experimental Social Psychology 21(2): 161-177.

JoHANsson, M. (2007): «Represented discourse in answers. A cross-cultural perspective on French and British political interviews» en FETzer, A.; G. E. Lauerbach, G. (2007): Political discourse in the media, Amsterdam, John Benjamins Publishing.

López, E. A.; J. De SANTiago (2000): Retórica y comunicación política, Madrid, Cátedra.

Martín Rojo, L.; E. Alcalá; A. Garí; L. Mijares; I. Sierra; M. A. RodríGUEZ (2003): ¿Asimilar o integrar? Dilema ante el multilingüismo en las aulas, CIDE.

Martín Rojo, L.; T. A. Van DiJK (1998): «Había un problema, y se ha solucionado. Legitimación de la expulsión de migrantes ilegales en el discurso del Parlamento Español» en Martín Rojo, L.; R. WhitTaKer (eds.) (1998): Poder-decir o el poder de los discursos, Madrid, Arrecife UAM. 169-234.

Moscovici, S. (1979): El psicoanálisis su imagen y su público, Buenos Aires, Huemul.

- (1986): Psicología social, Barcelona, Paidós.

- (2001): «Why a Theory of Social Representation?», en DEAux, K.; G. Philogène (eds.) (2001): Representations of the social. Bringing theoretical traditions, Malden, Blackwell. 8-35.

MusolfF, A. (2000): «Political imagery of Europe: A house without exit doors?», Journal of multilingual and multicultural development, 21 (3): 216-229.

Piaget, J. (1977): Estudios sociológicos, Barcelona, Ariel.

RaYa Lozano, E. (1999): «Notas para el análisis de los problemas y las políticas de inmigración en España» Intervención psicosocial: Revista sobre igualdad y calidad de vida, 8 (2):143-162.

ReEves, F. (1983): British Racial discourse, A study of British political discourse about race and race-related matters, Cambridge, Cambridge University Press.

Ribas, M. (2005): «Les metàfores de l'exclusió (Una reflexió sobre discurs i cognició social)» en Otal, J. L.; I. Navarro; B. Bellés (eds.) (2005): Cognitive and Discourse approaches to metaphor and metonymy, Castellón, Universitat Jaume I. 279-287.

RYan, W. (1976): Blaming the Victim, New York, Vintage.

SaChetTi, E.; J. J. Trigo (2009): El discurso político español sobre inmigración. Análisis crítico, Sevilla, Andalucía Acoge. 
Santamaría, E. (2002): «Inmigración y Barbarie. La construcción social y política del inmigrante como amenaza», Papers: revista de sociología, 66: 5975.

Simon-Vandenbergen, A. M.; P. R. White; K. Aijmer (2007): «Presupposition and 'taking for granted' in mass communicated political argument» en Fetzer, A.; G. E. Lauerbach (eds.) (2007): Political discourse in the media, Amsterdam, John Benjamins Publishing. 31-74.

Slomp, H. (2000): European Politics into the Twenty-First Century: Integration and Division, Westport, Praeger.

ToRrens, X. (2002): El racismo cultural en las democracias contemporáneas, tesis doctoral no publicada, Barcelona, Universitat de Barcelona.

VAN DiJK, T. A. (1977): «Context and cognition», Journal of pragmatics, 1: 211232.

- (1984): Prejudice in discourse: An analysis of ethnic prejudices in cognition and conversation, Amsterdam, John Benjamins.

- (1993): «Discourse and cognition in society», en Crowley, D.; D. Mitchell (eds.) (1993): Communication Theory Today, Oxford, Pergamon Press. 107-126.

- (1995): «Discourse analysis as ideology analysis», en SCHÄFFNER, C. ; A. Wenden (eds.) (1995): Language and Peace, Aldershot, Dartmouth Publishing. 17-33.

- (1997): «Cognitive context models and discourses», en STAMEnow, M. (ed.) (1997): Language Structure, Discourse and the Access to Consciousness, Amsterdam, John Benjamins. 189-226.

- (1998): Ideology: A multidisciplinary approach, London, Sage.

- (2000): «Ideologies, Racism, Discourse: Debates on immigration and ethnic issues», en WAL, J.; M. VerkuYten (eds.) (2000): Comparative perspectives on racism, Aldershot, Ashgate. 91-116.

- (2001 a): «Multidisciplinary CDA: a plea for diversity», en WoDAK, R.; M. MEYER (eds.) (2001 a): Methods of Critical Discourse analysis, London, Sage. 95-120.

- (2001 b): «Discourse, Ideology and Context», Folia lingustica XXX/1. 2: 11-40.

- (2002 a): «Political discourse and political cognition», en Chilton, P.; C. SCHÄFFner (eds.) (2002 a): Politics as text and talk: analytic approaches to political discourse, Amsterdam, John Benjamins. 204-236.

- (2002 b): «Political Discourse and Ideology», en LORDA, C. U.; M. RIBAS (eds.) (2002 b): Anàlisi del discurs polític, Barcelona, Universitat Pompeu Fabra/IULA. 15-34. 
- (2003 a): «The discourse-knowledge interface», en WEISS, G.; R. WODAK (eds.) (2003 a): Critical Discourse Analysis. Theory and Interdisciplinarity, Houndsmills, Palgrave-MacMillan. 85-109.

- (2003 b): Ideología y Discurso, Barcelona, Ariel.

- (2003 c): Dominación étnica y racismo discursivo en España y Latino América, Barcelona, Gedisa.

- (2007): «Ideology and Discourse Analysis», en Freeden, M. (ed.) (2007): The meaning of ideology. Cross-disciplinary perspectives, London, Routledge. 110-136.

- (2007 b) (coord.): Racismo y Discurso en América Latina, Barcelona, Gedisa.

- (2008): Discourse and Context: A sociocognitive Approach, Cambridge, Cambridge University Press.

- (2009): «Critical Discourse Studies: A Socio-cognitive Approach», en R. WodAK; M. Meyer (eds.) (2009): Methods of Critical Discourse Analysis, London, Sage. 62-106.

Van Leeuwen, T. (1995): «Representing Social Action», Discourse \& Society, 6 (81): 81-106.

- (1996): «The representation of social actors», en C. R. CaldasCoulthard; M. Coulthard (eds.) (1996): Texts and Practices - Readings in Critical Discourse Analysis, New York, Routledge.

Worchel, S.; J. COOPer; G. Goethals; J. Olson (2002): Psicología social, México, Thomson.

Zapata-Barrero, R.; E. González; E. SánChez (2008): El discurso político en torno a la inmigración en España y en la Unión Europea, Madrid: Ministerio de Trabajo e Inmigración, Subdirección general de información.

Zapata-Barrero, R.; T. A. VAN DiJK (2007): Discursos sobre la inmigración en España, Barcelona, Fundació Cidob. 